\title{
Instanton-Induced Polarization in Exclusive Hyperon Photoproduction
}

\author{
Nikolai Kochelev, ${ }^{a, 1]}$ \\ (a) School of Physics and Astronomy, Seoul National University, \\ Seoul 151-747, Korea \\ (b) Bogoliubov Laboratory of Theoretical Physics, Joint Institute for Nuclear Research, \\ Dubna, Moscow region, 141980 Russia
}

\begin{abstract}
It is shown that instantons can provide an explanation of the strong polarization transfer observed in exclusive hyperon photoproduction at JLab.
\end{abstract}

${ }^{1}$ kochelev@theor.jinr.ru 
In recent exclusive hyperon photoproduction experiments at JLab a remarkably strong transfer of polarization from the initial photon to the final hyperon has been observed in the reactions $\gamma+p \rightarrow K^{+}+\Lambda$ and $\gamma+p \rightarrow K^{+}+\Sigma^{0}$ [1, 2]. The QCD mechanism underlying this polarization transfer remains unexplained; to date, only a qualitative, phenomenological explanation has appeared in the literature [3], which is based on an ad hoc assumption of complete spin transfer from the photon to the strange quark. In this Brief Report we discuss a possible instanton-based mechanism for the strong correlation observed between the helicities of the initial photon and final hyperon.

Instantons, strong vacuum gluon fields which describe sub-barrier transitions between topologically different vacua in QCD, are known to play an important rôle in the dynamics of the strong interaction (for a review see [4]). In particular, instantons induce the 't Hooft four-quark interaction, which for massless quarks is given by [5]

$$
\begin{aligned}
\mathcal{H}^{\prime} t_{\text {Hooft }} & =\int d \rho n(\rho)\left(\frac{4}{3} \pi^{2} \rho^{3}\right)^{2}\left\{\bar{u}_{R} u_{L} \bar{s}_{R} s_{L}\left[1+\frac{3}{32}\left(1-\frac{3}{4} \sigma_{\mu \nu}^{u} \sigma_{\mu \nu}^{s}\right) \cdot \lambda_{u}^{a} \lambda_{s}^{a}\right]\right. \\
& +(u \rightarrow d)+(s \rightarrow d)+(R \rightarrow L)\}
\end{aligned}
$$

where $n(\rho)$ is the instanton density and $\rho$ is the instanton size. Here we shall assume that the instanton-induced interaction (1) is responsible for the observed strong correlation between the chiralities of the initial and final quarks.

The interaction (1) specifies that the initial quarks interacting through the instanton (anti-instanton) have left-handed (right-handed) chiralities, and that the final quarks have the opposite right-handed (left-hand) chiralities. For massless quarks chirality coincides with helicity, so we anticipate fully correlated initial and final helicities for the quarks interacting through the instanton field.

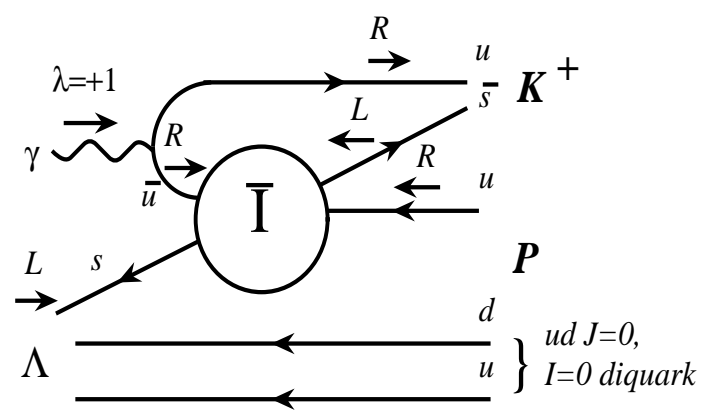

(a)

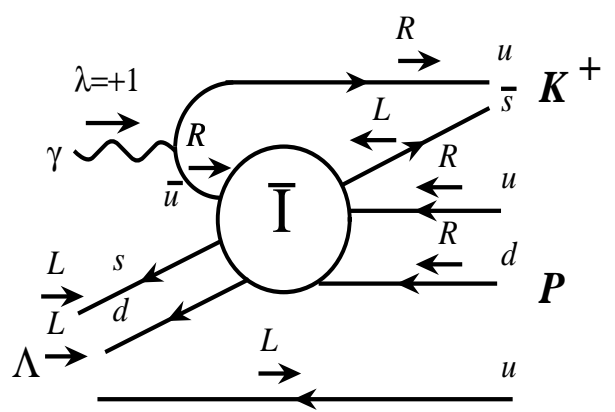

(b)

Figure 1: The instanton mechanism for polarization transfer to a $\Lambda$ hyperon: a) fourquark and b) six-quark instanton induced interaction contributions. Here I denotes the instanton, $\mathrm{R}$ and $\mathrm{L}$ are the quark helicities, and the detached arrows show the directions of the quark spins.

For definiteness we consider exclusive instanton-induced $\Lambda$ photoproduction due to interaction (1) in the center of mass system (Fig.1a). We emphasize that the same coordinate system was been used by the JLab experiment [1, 2] for analysis of the photon to $\Lambda$ helicity transfer. Inspection of the figure shows that the helicities of the initial light 
quarks which couple to the photon will be opposite to the helicities of the final $s \bar{s}$ quark pair. Since the polarization of the $\Lambda$-hyperon is given by the polarization of the $s$ quark (in the valence approximation), the $\Lambda$ should be fully polarized in the direction of the photon helicity 1 We note in passing that there is an additional instanton contribution to this reaction due to the six-quark instanton-induced interaction (Fig.1b). Although this additional contribution leads to the same strange quark helicity orientation relative to the photon chirality as the four-quark interaction (1), it should be strongly suppressed in scale. This six-quark interaction requires a double quark spin flip (of the d- and s-quarks in Fig.1b); one cannot combine the final quarks to form a spin-1/2 hyperon in the nonrelativistic limit. It is also clear that the one-instanton mechanism may only be applicable at rather low value of energy excess $Q$ above threshold. $\left(Q=W-M_{\Lambda}-M_{K} \leq M_{S}\right.$, where $M_{S} \approx 2 \mathrm{GeV}$ is the so-called sphaleron energy, which is the height of the potential barrier between different vacua [6]. At higher energies, multiinstanton contributions should lead to a decorrelation of hyperon and photon helicities. Due to the suppression of multiinstanton contributions at large photon virtuality, one might anticipate that the one-instanton approximation is better justified in exclusive electroproduction, in which a strong correlation between photon and hyperon helicity has also been observed [7]. In this case however one should carefully separate the zero-helicity longitudinal photon contribution.

In our conjecture the valence quark approximation for hadron and photon wave functions is using. It is evident that sea quark contributions should reduce helicity correlations at high energy. In the context of the instanton model of the QCD vacuum, these non-valence components in hadron state vectors are related to the admixture of quarkantiquark pairs and gluons created by instantons. This effect is not incorporated in our single-instanton model of hyperon production at energies not far above threshold.

The validity of this instanton mechanism for polarization transfer can be tested through studies of exclusive hyperon photoproduction in association with a vector meson $\left(K^{*}\right)$. In this case we anticipate fully longitudinal polarization of the $K^{*}$, due to the quark chirality flip at the instanton vertex (see Fig.1).

In summary, we have suggested a new mechanism for polarization transfer in exclusive hyperon photoproduction, based on 't Hooft's instanton-induced four-quark interaction. This mechanism can be tested through studies of hyperon photoproduction associated with a strange vector $\left(K^{*}\right)$ meson.

We are happy to acknowledge useful discussions of this work with A.E.Dorokhov. The author is very grateful to the School of Physics and Astronomy of Seoul National University, and especially Prof. Dong-Pil Min, for their kind hospitality. This work was supported in part by the Brain Pool program of the Korea Research Foundation through KOFST grant $042 \mathrm{~T}-1-1$.

\section{References}

[1] R. Bradford and R. A. Schumacher [CLAS Collaboration], arXiv:nucl-ex/0611034.

\footnotetext{
${ }^{1}$ The case of $\Sigma^{0}$ photoproduction is more complicated because the spin state of this hyperon is not determined by the strange quark alone.
} 
[2] P. Ambrozewicz, D. S. Carman, R. J. Feuerbach, M. D. Mestayer, B. A. Raue, R. A. Schumacher and A. Tkabladze, arXiv:hep-ex/0611036.

[3] R. Schumacher, arXiv:nucl-ex/0611035.

[4] T. Schäfer and E. V. Shuryak, Rev. Mod. Phys. 70, 323 (1998) arXiv:hep-ph/9610451.

[5] M. A. Shifman, A. I. Vainshtein and V. I. Zakharov, Nucl. Phys. B 165, 45 (1980).

[6] R. A. Janik, E. Shuryak and I. Zahed, Phys. Rev. D 67, 014005 (2003)

[7] D. S. Carman et al. [CLAS Collaboration], Phys. Rev. Lett. 90, 131804 (2003) 